\title{
BMJ Open Effect of early versus standard central line removal on growth of very low birthweight premature infants: a protocol for a non-inferiority randomised controlled trial
}

\author{
Justyna Romańska, ${ }^{1}$ Wojciech Margas, ${ }^{2}$ Renata Bokiniec, ${ }^{3}$ Paweł Krajewski, ${ }^{1}$ \\ Joanna Seliga-Siwecka ${ }^{3}$
}

To cite: Romańska J, Margas W, Bokiniec R, et al. Effect of early versus standard central line removal on growth of very low birthweight premature infants: a protocol for a non-inferiority randomised controlled trial. BMJ Open 2019;9:e030167. doi:10.1136/ bmjopen-2019-030167

- Prepublication history and additional material for this paper are available online. To view these files, please visit the journal online (http://dx.doi. org/10.1136/bmjopen-2019030167).

Received 01 March 2019 Revised 14 August 2019 Accepted 20 August 2019

Check for updates

(c) Author(s) (or their employer(s)) 2019. Re-use permitted under CC BY-NC. No commercial re-use. See rights and permissions. Published by BMJ.

${ }^{1}$ Division of Neonatology and Neonatal Intensive Care Unit of First Department of Obstetrics and Gynecology, Medical University of Warsaw, Warszawa, Poland

${ }^{2} \mathrm{HTA}$ Consulting, Cracow, Poland ${ }^{3}$ Neonatal and Intensive Care Department, Medical University of Warsaw, Warszawa, Poland

Correspondence to

Dr Joanna Seliga-Siwecka; joanna.seliga@wum.edu.pl

\section{ABSTRACT}

Introduction Uncertainty exists regarding the optimal time for removal of central lines used to provide parenteral nutrition in preterm infants. The aim of this study is to determine whether earlier central line removal is noninferior to its removal after reaching full enteral intake, in respect to growth outcome of preterm infants.

Methods and analysis Very low birthweight premature infants will be recruited. Eligible infants will be randomised in equal proportions between two groups. In the intervention group central lines will be removed when infants reach $100 \mathrm{~mL} / \mathrm{kg} /$ day of enteral intake. In the control group central lines will be removed when infants reach $140 \mathrm{~mL} / \mathrm{kg} /$ day of enteral intake (full enteral intake). The primary outcome measure will be the difference between the two groups in weight at 36 weeks' postmenstrual age. Non-inferiority will be declared if the mean weight of children in the intervention group will be no worse than the mean weight of children from the control group, by a margin of $-210 \mathrm{~g}$.

Ethics and dissemination The Bioethics Committee of the Medical University of Warsaw approved the study protocol prior to recruitment. The findings of this trial will be submitted to a peer-reviewed journal (neonatology, paediatrics or nutrition). Abstracts will be submitted to relevant national and international conferences.

Trial registration number NCT03730883.

Protocol version Version 3. 14.08.2019.

\section{INTRODUCTION}

Over the last decades survival rates among very low birthweight (VLBW) infants have improved substantially. Beyond the risk of death and short-term morbidities, extreme prematurity results in an increased risk of long-term disabilities, which affect the quality of life among VLBW survivors and present a major public health issue. ${ }^{12}$ Optimising longterm outcome associated with prematurity is therefore the area of great interest in modern neonatology. Postnatal growth failure is one of the most common morbidities affecting
Strengths and limitations of this study

- A precise clinical question has been posed to fill a gap in knowledge: Is removing a central line prior to achieving full enteral intake safe, in respect to growth outcome of preterm infants?

- The intervention is simple and limited to a specific time point, which should promote adherence to the study protocol.

- The study protocol complies with the Standard Protocol Items: Recommendations for Interventional Trials statement.

- The primary outcome of the study is relatively short term and it may not reflect a longer term effect of the intervention if any.

- The non-inferiority margin may be questioned as it was arbitrarily set at $210 \mathrm{~g}$ after analysing epidemiological data from our hospital.

VLBW infants. It is defined as weight $<10$ th percentile at 36 weeks' postmenstrual age (PMA) or at discharge. ${ }^{3}$ Although by definition it is limited to early postnatal life, the effect of growth faltering often persists into early childhood and even later. ${ }^{2}$ It has been proven by numerous studies that the risk of postnatal growth failure is inversely related to birth weight. ${ }^{4}$ In a review written by Dusick et al, the authors stated that among a cohort of extremely low birthweight (ELBW) infants cared for at centres participating in the National Institute for Child and Human Development Neonatal Research Network during 2000-2001, 16\% of infants were identified as growth restricted at birth, while at 36 weeks' PMA, $89 \%$ of infants experienced growth failure. ${ }^{4}$ A positive relationship between nutritional support, growth and neurodevelopmental outcome is well documented in a number of trials. Stephens et al 
demonstrated that for each $10 \mathrm{kcal} / \mathrm{kg} /$ day increase in energy intake and each $1 \mathrm{~g} / \mathrm{kg} /$ day increase in protein intake during the first week of life, there was an associated 4.6 point increase and 8.2 point increase in Bayley Mental Developmental Index at 18 months' corrected age (CA), respectively. ${ }^{2}$ Furthermore, Ehrenkranz and coworkers reported, that as the velocity of weight gain and head circumference growth increased, the incidence of severe neurodevelopmental impairment decreased, among ELBW infants during their stay at the neonatal intensive care unit (NICU) ${ }^{5}$ Early aggressive nutrition is a standard of care in VLBW infants nowadays. This strategy is considered safe and may be beneficial in improving growth of premature infants. ${ }^{6}$ Adequate nutrition support in the first weeks of life is based mainly on parenteral nutrition that can be provided via central line (CL) access only. Thus, modern intensive care of the newborn cannot be implemented without using CLs that secure reliable intravenous access for weeks. On the other hand, CL insertion is an invasive procedure that puts a neonate at risk of mechanical and infectious complications. Central line-associated bloodstream infections (CLABSI) are the most common type of hospital-acquired infections in neonates. ${ }^{7}$ Such infections result in higher mortality rates and exert a detrimental effect on short-term morbidity and long-term neurodevelopment among survivors. ${ }^{7}$ Stoll et al demonstrated an association between bloodstream infections in preterm neonates and increased risk of poor neurodevelopmental outcome, including significantly lower mental and psychomotor Bayley Scales of Infant Development II scores, vision and hearing impairment and cerebral palsy. ${ }^{8}$ Coagulase-negative staphylococcus (CONS) is the most frequent pathogen responsible for CLABSI. Although the mortality rate of CONS sepsis is low, there is an increased risk of cognitive delay among survivors. ${ }^{9}$ In addition, CLABSIs prolong hospitalisation and result in significant increase in hospital costs. ${ }^{7011}$ With recognition of all the negative consequences of CLABSIs mentioned above, an effort to prevent such infections cannot be overstated. Current guidelines for preventing CLABSIs consist of a group of evidence-based practices called the CL bundle. ${ }^{112}$ The key components of the CL bundle include hand hygiene, maximal barrier precaution during insertion, chlorhexidine skin antisepsis, optimal catheter-site selection and prompt removal of an unnecessary line. The daily review of the need for a CL comes from an assumption that catheter dwell time is an important risk factor for CLABSI. This hypothesis has already been proven by a number of studies conducted in neonates. ${ }^{13} 14$ In the vast majority of VLBW infants, while parenteral nutrition is provided, enteral feedings are started and advanced until full enteral intake is achieved. There is a point in time where the advantages of continued parenteral nutrition are outweighed by the risk of prolonged central vascular access and infection. To our knowledge, there is only one randomised controlled trial that addresses the issue of discontinuation of parenteral nutrition and removing of CLs. In the study, VLBW infants were assigned to have CLs removed and parenteral nutrition discontinued at $100 \mathrm{~mL} / \mathrm{kg}$ / day vs $140 \mathrm{~mL} / \mathrm{kg} /$ day. Early CL removal resulted in a delay in time taken to regain birth weight, which was the primary outcome in the study.$^{15}$ In the absence of evidence from randomised trials regarding the issue of CL removal, recent guidelines are based mainly on experts' opinions and tend to vary considerably. Currently, many quality improvement initiatives targeted at reducing the rate of CLABSI incorporate guidelines for CL necessity and removal into the CL bundle. The California Perinatal Quality Care Collaborative and the Perinatal Quality Collaborative of North Carolina (PQCNC) suggest removing CLs when an infant reaches $>120 \mathrm{~mL} / \mathrm{kg} /$ day of enteral nutrition. ${ }^{16}{ }^{17}$ The PQCNC CLABSI project resulted in the largest reduction in CLABSI rate among different NICU collaboratives. The authors stated that standardised practice of timely CL removal was one of the key factors in CLABSI reduction. ${ }^{16}$ In spite of existing guidelines, significant diversity in practice among neonatologists and among different NICUs still exists. ${ }^{16}$ This results from the perception that earlier CL removal conflicts with optimal nutrition and increases the risk of suboptimal growth. There is a gap in knowledge regarding the safety of early CL removal in respect to growth outcome. Therefore, we decided to conduct a study in order to determine whether earlier CL removal is non-inferior to its removal after reaching full enteral intake in respect to weight of preterm infants at 36 weeks' PMA.

\section{METHODS AND ANALYSIS}

The study protocol closely follows reporting guidelines included in the Standard Protocol Items: Recommendations for Interventional Trials statement. ${ }^{18}$

\section{Objectives}

The primary objective of the trial is to determine whether earlier CL removal is non-inferior to its removal after full enteral intake is achieved, in respect to weight of preterm infant at 36 weeks' PMA. Secondary objectives include the influence of the intervention on the incidence of all-cause death, the incidence of CLABSI, time to regain birth weight, growth parameters at 36 weeks' PMA and at the age of 18 months' CA.

\section{Trial design}

The study is designed as a non-inferiority randomised controlled trial with two parallel groups. A stratified randomisation will be performed using permutated blocks of varying sizes with a 1:1 allocation.

\section{Study setting}

The study started in January 2019 at the neonatal department (a tertiary care clinical hospital for women: Neonatal and Intensive Care Department, The Medical University of Warsaw, Poland) with approximately 100 VLBW infants cared for annually. Providing parenteral nutrition via CL 
is standard of care used in a majority of these patients. In view of low rate of recruitment we included a second neonatal department (Division of Neonatology and Neonatal Intensive Care, First Department of Obstetrics and Gynaecology, The Medical University of Warsaw, Poland) in March 2019. We plan to include two more neonatal departments in the following 6 months. The enrolment period will extend over 24 months (January 2019 to January 2021).

\section{Eligibility criteria}

Caregivers must provide written informed consent before any study procedures occur.

At study inclusion patients must comply with all of the following criteria: (1) birth weight $\leq 1500 \mathrm{~g}$, (2) birth weight $\geq 3$ rd percentile at a given gestational age, (3) CL inserted (PICC, Peripherally Inserted Central Catheter or UVC, Umbilical Venous Catheter), (4) oral intake not exceeding $100 \mathrm{~mL} / \mathrm{kg} /$ day at randomisation, and (5) lack of congenital illness or malformation that may affect growth.

The exclusion criteria will be as follows: (1) birth weight $>1500 \mathrm{~g}$, (2) birth weight $<3$ rd percentile at a given gestational age, (3) no CL inserted, (4) oral intake $\geq 100 \mathrm{~mL}$ / $\mathrm{kg}$ /day at randomisation, (5) congenital illness or malformation that may affect growth, and (6) participation in other intervention trials that may affect primary outcome.

\section{Intervention}

Eligible infants will be randomised in equal proportions between two groups. In the intervention group (early CL removal) CLs will be removed at the time the infants reach $100 \mathrm{~mL} / \mathrm{kg} /$ day of enteral intake. In the control group (standard CL removal) CLs will be removed at the time the infants reach $140 \mathrm{~mL} / \mathrm{kg} /$ day of enteral intake (full enteral intake). CLs will be removed after three consecutive well-tolerated feedings with no contraindications for CL removal present such as:

- Necessity of administration of drugs that must be given via central venous access.

- Necessity of administration of drugs that must be given intravenously along with difficulties to secure peripheral venous access.

- Necessity of prolonged (>7 days) administration of drugs that must be given intravenously.

- Decision to continue parenteral nutrition along with difficulties to secure peripheral venous access.

Assessment of feeding tolerance will be at discretion of the physician taking care for the infant. After CL removal, infants in the intervention group may continue to receive parenteral nutrition via peripheral venous access depending on the decision of the physician. The solution used to provide parenteral nutrition via peripheral venous access will contain at maximum $2.5 \%$ amino acids, $10 \%$ glucose and no calcium or phosphate preparations to ensure the fluid's osmolality does not exceed $900 \mathrm{mOsm} / \mathrm{L}$ and that the solution will be well tolerated when administered via peripheral vein.
Parenteral nutrition will be prescribed according to the local protocol.

Enteral nutrition will be initiated during the first days of life and advanced gradually at the discretion of the physician.

\section{Criteria for discontinuing allocated intervention}

If an infant presents with a serious complication of central venous catheterisation such as CLABSI, pleural or pericardial effusion, cardiac tamponade, ascites, pericarditis or catheter-related thrombosis and requires earlier CL removal, this will be done regardless of the study protocol. The assigned intervention will be discontinued, unless a second CL is inserted. Excluded patients will be retained in the study to enable data collection and management. The infants' data will be included in the statistical analysis to compare the rate of CLABSI between two study groups. If an infant is diagnosed with a disease known to significantly impair growth (ie, short bowel syndrome) he will be excluded from the study.

\section{Compliance}

Medical records (MR) of patients included in the study will be visibly marked to promote adherence to the study protocol. A flow chart explaining inclusion, exclusion and discontinuation criteria will be available in the patient's MR. Before starting the study, a meeting will be scheduled to introduce the staff to the study protocol. This meeting will include:

- Brief presentation of the study protocol including justification for undertaking the trial and possible impact of the study outcome on everyday practice.

- Instructions about the way the intervention should be applied.

Face-to-face adherence reminder sessions will take place on a monthly basis at the department where the study will be conducted. Staff will be asked about any problems they are experiencing with implementing the study.

\section{Explanation for choice of comparators}

In the intervention group, we chose to discontinue CLs at $\geq 100 \mathrm{~mL} / \mathrm{kg}$ /day of oral feeds, following a common conception, that this reduces the rate of CLABSI. In the control group, we chose to remove CLs at $\geq 140 \mathrm{~mL} / \mathrm{kg}$ / day of oral feeds (full enteral intake), as this is considered the standard of care in the study site. Its selection as comparator is therefore justified.

\section{Outcome measures}

The primary outcome measure will be weight at 36 weeks' PMA. Non-inferiority will be declared if a lower bound of the $95 \% \mathrm{CI}$ around the estimated mean difference in weight at 36 weeks' PMA between the two intervention arms lies above the non-inferiority margin set at $-210 \mathrm{~g}$.

Secondary outcomes will include:

- Head circumference at 36 weeks' PMA.

- Length at 36 weeks' PMA.

- The rate of CLABSI within the intervention period and 2 days after completion. 
Table 1 The timeline of the study

\begin{tabular}{|c|c|c|c|c|c|}
\hline & vo & V1 & V2 & V3 & V4 \\
\hline Study plan & At birth & 0-14 days & $\begin{array}{l}\geq 100 \mathrm{~mL} / \mathrm{kg} \text { of enteral intake versus } \\
\geq 140 \mathrm{~mL} / \mathrm{kg} \text { of enteral intake }\end{array}$ & $\begin{array}{l}36 \text { weeks' } \\
\text { PMA }\end{array}$ & $\begin{array}{l}18 \text { months' } \\
\text { CA }\end{array}$ \\
\hline Eligibility assessment & & $x$ & & & \\
\hline Informed consent reception & & $x$ & & & \\
\hline Anthropometry & $x$ & & & $x$ & $x$ \\
\hline Data collection & $x$ & & & & \\
\hline
\end{tabular}

CA, corrected age; PMA, postmenstrual age.

- Growth parameters (weight, length and head circumference) at 18 months' CA.

- All-cause death.

- Time to regain birth weight.

- Number of peripheral intravenous accesses inserted until discontinuation of parenteral nutrition provided after CL removal.

- Need for CL insertion within 7 days following intervention because of feeding intolerance.

- Length of hospital stay.

\section{Participant timeline}

The intervention period, in terms of data necessary for the primary outcome, will last until infants reach 36 weeks' PMA.

Anthropometric measurements (weight, length and head circumference) will be collected on three separate occasions, that is, at birth, at 36 weeks' PMA and at 18 months' CA.

The timeline of the study is presented in table 1.

\section{Sample size}

Based on retrospective epidemiological data of approximately 117 VLBW neonates retrieved from the MRs of the Neonatal and Intensive Care Department of the Medical University of Warsaw, the estimated mean (SD) weight at 36 weeks' PMA or at the day of discharge was 2096 (357) g. Equal value of SD was assumed for the sample size estimation for both groups in the present study. However, due to earlier cessation of feeding through CL compared with the control group, growth rate in the intervention group could be impaired until full enteral intake is reached. This leads to an increased risk of failure to demonstrate non-inferiority, and consequently inflates the minimum sample size required to reach test validity. As a standard hospital practice, after CL removal neonates receive parenteral nutrition via peripheral venous access when needed, which may partially counterbalance growth slowdown after earlier cessation of parenteral nutrition provided by CL. The sample size was estimated to detect a putative $50 \mathrm{~g}$ mean weight difference between both groups, assessed by the non-inferiority test for two independent means $(\alpha=0.025 \%$ and $80 \%$ power). The non-inferiority margin was set at $-210 \mathrm{~g}$, which represents a $10 \%$ decrease in mean body weight estimated from retrospective epidemiological data. Moreover, the value of $210 \mathrm{~g}$ is within one centile range. Assuming equal sized groups and the $20 \%$ dropout rate, the number of subjects to be recruited to each group will be 99 . Sample size calculations were performed with a cost-free available power and sample size calculator provided at http://powerandsamplesize. com/Calculators (HyLown Consulting, USA).$^{19}$

\section{Sequence generation}

Participants will be randomly assigned to either the intervention or control group with a 1:1 allocation as per a computer-generated randomisation schedule.

A separate randomisation list will be created for each stratum defined as the cross-classification of two balancing factors: gender (girls vs boys) and gestational age $(\leq 26$ weeks +6 days vs $\geq 27$ weeks +0 days).

The allocation sequence will be generated by the service managed by an external statistical team. The randomisation schedule will remain with the statistical team for the whole duration of the study, investigators will be blinded to block lengths; however, sufficiently short lengths will be used to minimise the possible imbalance between groups. This adopted strategy complies with the International Conference on Harmonisation (ICH) E9 Guidance on statistical principles for clinical trials. ${ }^{20}$

\section{Allocation concealment mechanism and implementation}

Allocation concealment will be ensured, as the statistical team will not release the randomisation code until the patient has been recruited into the trial, which takes place after all baseline measurements have been completed. Randomisation will be requested by one of the recruitment staff. The information about treatment allocation will then be given to the staff taking care of the patient. The staff will be aware of the past treatment allocations. In order to reduce the risk of predictability and selection bias, variable block sizes will be chosen at random from a specified subset of block lengths. 


\section{Blinding}

Due to the nature of the intervention, staff cannot be blinded to allocation.

\section{Data collection and management}

All patients' data will be collected using an electronic case report form. Access to the study data will be restricted to involved researchers only. A password system will be used to control access.

Researchers will be responsible for obtaining anthropometric measurements. In order to perform accurate, precise and standardised measurements they will follow guidelines from the Anthropometry Handbook prepared by the INTERGROWTH-21 (st) Anthropometry Group. ${ }^{21}$

Daily protein, glucose, lipid and energy intakes will be collected by chart review during provision of parenteral nutrition. Data regarding daily enteral intakes for the first 4 weeks of life will be collected. Mean daily protein and energy intakes will be calculated for weeks 1, 2, 3 and 4 and presented as the amount received per kilogram of body weight per day. Moreover, the data on the type of enteral feeding at discharge will be collected.

CLABSI will be defined according to the Centers for Disease Control and Prevention/National Healthcare Safety Network criteria. The CLABSI rate per 1000 CL-days will be calculated by dividing the number of CLABSIs by the number of CL-days and multiplying the result by $1000 .^{22}$

In infants in whom only one blood culture will be obtained, which turns out positive for a common skin commensal, we will apply criteria from Vermont Oxford Network to classify the event as a bloodstream infection.

In order to determine the day of regaining birth weight we will follow the definition used in the study conducted by Drenckpohl et al. If the infants remain above their birth weight for 3 consecutive days, the first day of the three weights will be used as the date of regaining birth weight. $^{23}$

These data will be obtained from the patients' MR.

Randomised infants prematurely discontinued from the study will have the same clinical evaluations performed as if they remained in the study. All participants will be included in the intention-to-treat (ITT) analysis, regardless of adherence. In addition, per-protocol (PP) analysis of patients whose observations have been completed in accordance with the protocol will be performed to estimate the effect of missingness or protocol deviations on the statistical analysis. The analysis for both, ITT and PP population, is advised in non-inferiority trials according to ICH E9 Note for Guidance and The European Agency for the Evaluation of Medicinal Products guidance, as both ITT and PP play different roles in the analysis.

\section{Statistical methods}

Baseline characteristics will be presented for all children in the ITT and PP analysis sets, by the treatment group. Dichotomous and nominal variables will be presented as frequencies, ordinal and discrete variables as a median and $\mathrm{IQR}$ and continuous variables as a mean and $\mathrm{SD}$, along with $95 \%$ CI. In case of missing data that is, due to adverse events (AE) or dropouts, in the follow-up analysis a weighted average to take account of changes in the gender ratio will be applied. Continuous variables will be tested against normality of distribution and the equality of variances between groups. For continuous variables distributed normally the differences in means will be tested using t-test, for continuous variables not distributed normally and for discrete and ordinal variables between groups comparison will be performed using Mann-Whitney U test. The primary outcome will be assessed with one-tailed unpaired two-sample t-test for non-inferiority. Secondary and safety outcomes will be tested for two-sided superiority. Proportions of dichotomous variables will be tested using $\chi^{2}$ or Fisher's exact test, whichever is appropriate. In addition, the risk ratio (RR), the OR and the number needed to treat will be assessed. For secondary outcomes, the difference will be considered significant when $\mathrm{p}$ values calculated in statistical tests will be $<0.05$ or when the $95 \%$ CI for the mean difference will not include 0 , or 1 in case of RR or OR.

\section{Monitoring and harms}

Data monitoring committee has not been established since the intervention within the trial does not differ from standard of care in VLBW infants.

We will define an $\mathrm{AE}$ as any untoward medical occurrence in a subject without regard to the possibility of a causal relationship. AEs will be collected after the subject has provided consent and enrolled in the study. All AEs occurring after entry into the study and until hospital discharge will be recorded. An $\mathrm{AE}$ that meets the criteria for a serious adverse event (SAE) between study enrolment and hospital discharge will be reported to the local ethical committee. An SAE for this study is any untoward medical occurrence that is believed by the investigators to be causally related to study intervention and results in any of the following: fatal or life-threatening condition, permanent disability or prolonged hospitalisation. SAEs occurring after a subject is discontinued from the study will not be reported unless the investigators feel that the event may have been caused by a protocol procedure.

The study will monitor for the following CL-related AEs: CLABSI, pleural or pericardial effusion, cardiac tamponade, ascites, pericarditis or catheter-related thrombosis. The list of anticipated CL-related AEs will be an integral part of the electronic questionnaire. The openended section 'other adverse events' will also be included in the questionnaire. The possibility of continuing parenteral nutrition by peripheral venous access is aimed to reduce the risk of dehydration and malnutrition attributed to earlier CL removal. Taking into account the possible AEs related to the peripheral venous access and the pain caused by its insertion we will collect data on the number of peripheral intravenous accesses inserted until discontinuation of parenteral nutrition and AEs related to its usage. We will monitor patients for the necessity of 
CL insertion because of feeding intolerance within 7 days following intervention. The data also will be noted in the electronic questionnaire.

The data on AEs will be presented for each study group of the trial and each type of AE separately. For events with many recurrences we will provide the number of affected participants and the number of events for each study group.

\section{Patient and public involvement}

Patients and public were not involved in the design of the study.

\section{Ethics and dissemination}

Any important modifications to the protocol including changes of study objective, study design, patient population, sample sizes or study procedure will be documented in the study protocol as formal amendments. Such amendments will be submitted to the Bioethics Committee of the Medical University of Warsaw for a review.

Patients eligible for the study will be offered to participate in the trial by one of the researchers. Parents considering participation will receive oral and written information about the study and they will be given the opportunity to ask questions. The researcher prior to enrolment in the study will obtain written consent from the patient's caregiver (see online supplementary appendix 1).

All individual participant data collected during the trial will be available, after deidentification. The study protocol will also be available. The above documents will be accessible to anyone who provides a methodologically sound proposal, immediately following the publication with no end date.

The findings of this trial will be submitted to a peer-reviewed journal (neonatology, paediatrics or nutrition). Abstracts will be submitted to relevant national and international conferences.

Acknowledgements Advice given by Tomasz Wawrzoniak has been a great help in planning the study and editing the protocol. We also thank Dr Tomasz Pomianek for proofreading the protocol.

Contributors JR and JSS conceptualised the study. WM contributed to the study design. JR, JSS and WM planned the statistical analysis. JR wrote the first draft of the protocol. RB and PK critically reviewed the protocol and accepted the final manuscript as submitted. All authors read and approved the final version.

Funding This work is supported by the Medical University of Warsaw internal departmental fund.

Competing interests None declared.

Patient consent for publication Obtained.

Ethics approval The Bioethics Committee of the Medical University of Warsaw approved the study protocol before recruitment started.

Provenance and peer review Not commissioned; externally peer reviewed.

Open access This is an open access article distributed in accordance with the Creative Commons Attribution Non Commercial (CC BY-NC 4.0) license, which permits others to distribute, remix, adapt, build upon this work non-commercially, and license their derivative works on different terms, provided the original work is properly cited, appropriate credit is given, any changes made indicated, and the use is non-commercial. See: http://creativecommons.org/licenses/by-nc/4.0/.

\section{REFERENCES}

1. Behrman R, Butler A. Preterm birth. Washington, D.C.: National Academies Press, 2007.

2. Stephens BE, Walden RV, Gargus RA, et al. First-week protein and energy intakes are associated with 18-month developmental outcomes in extremely low birth weight infants. Pediatrics 2009;123:1337-43.

3. Poindexter B. Approaches to growth faltering. World Rev Nutr Diet 2014;110:228-38.

4. Dusick AM, Poindexter BB, Ehrenkranz RA, et al. Growth failure in the preterm infant: can we catch up? Semin Perinatol 2003;27:302-10.

5. Ehrenkranz RA, Dusick AM, Vohr BR, et al. Growth in the neonatal intensive care unit influences neurodevelopmental and growth outcomes of extremely low birth weight infants. Pediatrics 2006;117:1253-61.

6. Ibrahim HM, Jeroudi MA, Baier RJ, et al. Aggressive early total parental nutrition in low-birth-weight infants. J Perinatol 2004;24:482-6.

7. Garber SJ, Puopolo KM. Prevention of central line-associated bloodstream infections among infants in the neonatal intensive care unit. Neoreviews 2015;16:e211-20.

8. Stoll BJ, Hansen NI, Adams-Chapman I, et al. Neurodevelopmental and growth impairment among extremely low-birth-weight infants with neonatal infection. JAMA 2004;292:2357-65.

9. Alshaikh B, Yee W, Lodha A, et al. Coagulase-Negative Staphylococcus sepsis in preterm infants and long-term neurodevelopmental outcome. J Perinatol 2014;34:125-9.

10. Donovan EF, Sparling K, Lake MR, et al. The investment case for preventing NICU-associated infections. Am J Perinatol 2013;30:179-84.

11. How-to guide: prevent central line-associated bloodstream infections. Cambridge, MA: Institute for healthcare improvement, 2012. Available: www.ihi.org

12. O'Grady NP, Alexander M, Dellinger EP, et al. Guidelines for the prevention of intravascular catheter-related infections. Infect Control Hosp Epidemiol 2002;23:759-69.

13. Milstone AM, Reich NG, Advani S, et al. Catheter dwell time and CLABSIs in neonates with PICCs: a multicenter cohort study. Pediatrics 2013;132:e1609-15.

14. Sengupta A, Lehmann C, Diener-West M, et al. Catheter duration and risk of CLA-BSI in neonates with PICCs. Pediatrics 2010;125:648-53.

15. Perrem LM SJ, O'Sullivan A, et al. Randomised controlled trial comparing PICC removal and parenteral nutrirtion discontinuation at $100 \mathrm{mls} / \mathrm{kg} /$ day versus $140 \mathrm{mls} / \mathrm{kg} /$ day enteral feeding in preterm infants less than 1500g. Eur J Pediatr 2016;175:1517.

16. Fisher D, Cochran KM, Provost LP, et al. Reducing central lineassociated bloodstream infections in North Carolina NICUs. Pediatrics 2013;132:e1664-71.

17. Nutritional support of the very low birth weight infant, 2008. Available: https://www.cpqcc.org/sites/default/files/NUTRITIONAL SUPPORT OF THE VLBW INFANT_- REVISED 2008EntireToolkit. pdf [cited 6 Feb 2019].

18. Chan A-W, Tetzlaff JM, Altman DG, et al. Spirit 2013 statement: defining standard protocol items for clinical trials. Ann Intern Med 2013;158:200-7.

19. HyLown Consulting LLC G. Compare 2 Means 2-Sample NonInferiority or Superiority | Power and Sample Size Calculators | HyLown [Internet]. Powerandsamplesize.com, 2018. Available: http:// powerandsamplesize.com/Calculators/Compare-2-Means/2-SampleNon-Inferiority-or-Superiority [cited 6 Feb 2019].

20. International Conference on Harmonisation, E-9 Document, Guidance on statisticalprinciples for clinical trials. Federal Register 1998;63:49583-49598, 1998. Available: http://www.fda.gov/cder/ guidance/91698.pdf

21. Cheikh Ismail L, Knight HE, Ohuma EO, et al. Anthropometric standardisation and quality control protocols for the construction of new, international, fetal and newborn growth standards: the INTERGROWTH-21st project. BJOG 2013;120(Suppl 2):48-55.

22. Cdc.gov. Ach surveillance for BSI (CLABSI) | NHSN | CDC, 2019. Available: https://www.cdc.gov/nhsn/acute-care-hospital/clabsi/ index.html [Accessed 6 Feb 2019].

23. Drenckpohl D, McConnell C, Gaffney S, et al. Randomized trial of very low birth weight infants receiving higher rates of infusion of intravenous fat emulsions during the first week of life. Pediatrics 2008;122:743-51. 